NIST Technical Note 1611

\title{
Fire-Front Propagation Using the Level Set Method
}

Ronald G. Rehm

Randall J. McDermott

QC National Institute of Standards and Technology - U.S. Department of Commerce 45753

1611

2009

c.2 



\section{NIST Technical Note 1611}

\section{Fire-Front Propagation Using the Level Set Method}

Ronald G. Rehm Randall J. McDermott U.S. Department of Commerce Building and Fire Research Laboratory National Institute of Standards and Technology

Gaithersburg, MD 20899-8663

March 2009 Issued April 2009

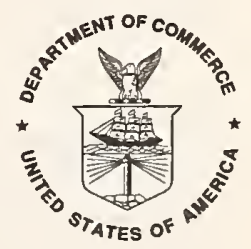

U.S. Department of Commerce Gary Locke, Secretary 
Certain commercial entities, equipment, or materials may be identified in this document in order to describe an experimental procedure or concept adequately. Such identification is not intended to imply recommendation or endorsement by the National Institute of Standards and Technology, nor is it intended to imply that the entities, materials, or equipment are necessarily the best available for the purpose.

National Institute of Standards and Technology Technical Note 1611

Natl. Inst. Stand. Technol. Tech. Note 1611, 15 pages (April 2009)

CODEN: NSPUE2 


\title{
Fire-Front Propagation Using the Level Set Method
}

\author{
Ronald G. Rehm and Randall J. McDermott \\ Building and Fire Research Laboratory \\ National Institute of Standards and Technology \\ Gaithersburg, MD 20899
}

March 25, 2009

\begin{abstract}
Propagation of an outdoor fire front in wildland or in a combination of wildland and structural fuels (the so-called wildland-urban interface or WUI fire), can be modelled as an initial-value problem using either a Lagrangian or an Eulerian description. The equations associated with each description are presented, and the methods used to solve the equations are discussed. Some comparisons between the two methods are also made. The emphasis in this report is on the Eulerian equations and on the level-set numerical method. Earlier studies had presented the Lagrangian formulation, and a method-of-lines solution. Advantages of the Eulerian/level-set method are discussed, and several examples that illustrate these advantages are presented.
\end{abstract}

\section{Introduction}

In earlier papers, [1]-[4], mathematical models for the propagation of wind-driven fire fronts in a combination of wildland and structural fuels, i.e., models for Wildland-Urban Interface (WUI) fires, have been presented and discussed. The fire front in the wildland fuel is often treated as a front of zero thickness and is tracked in a Lagrangian description. The initial front is spatially discretized into a set of nodes whose time evolution are determined as the solution of a set of ordinary differential equations (ODEs) by the method-of-lines (MOL). Results for the behavior of the front were reported for wind-blown fires in the presence of single or multiple burning structures on level or uneven terrain, Rehm [2] and Rehm and Mell [4].

The model presented in these papers, [1]-[4], is unique in that it attempts to describe the behavior of wind-blown WUI fires, not just wildland fires. Although Lagrangian models for front propagation in wildland fuels, i.e., wildfires, have been around for a few decades, no one has attempted to extend the methodology to WUI fires. In Section 2 of this report, for comparison, we summarize the Lagrangian formulation and its solution for wind-blown fire-front propagation presented in references [1]-[4]. Then, in Section 3, we present an Eulerian formulation of fire-front propagation and, in Section 4, a solution to these equations by the numerical technique known as the level-set method.

\section{Lagrangian Equations}

For simplicity in this section, we consider only fire-front propagation in the presence of burning structures over level ground (no topography). We follow the formulation presented in the Appendix of Rehm [2]. The governing equations are the ordinary differential equations (ODEs) describing the propagation 
of an element of the fire front in the horizontal plane:

$$
\frac{d \vec{R}}{d t}=(\vec{U} \cdot \vec{n}) \vec{n}
$$

The equations are given in vector form $\vec{R}=x \vec{i}_{x}+y \vec{i}_{y}$, where $\vec{i}_{x}, \vec{i}_{y}$ are unit vectors in the $\mathrm{x}$ and $\mathrm{y}$ directions. $\vec{U}=U_{x} \vec{i}_{x}+U_{y} \vec{i}_{y}$ is the spread rate (or the rate of spread (ROS) vector in $\mathrm{m} / \mathrm{s}$ ) of the fire front at the location $(x, y)$, and $n_{x}, n_{y}$ are the components of the unit normal to the fire front directed toward the unburnt fuel.

At each point, the fire front is advanced in the direction normal to the front at a speed determined by the local ROS for the fire. This ROS, in turn, can depend on several variables including the total wind speed at that location. Let $\vec{V}=V_{x} \vec{i}_{x}+V_{y} \vec{i}_{y}$ be the total wind velocity at a specified height. Assume that the linear relation for Australian grass, Cheney et al [9], given below, is valid for the normal ROS and the local normal wind velocity:

$$
U_{n}=\tilde{r}_{0}\left(1+c_{f} V_{n}\right)
$$

where $U_{n}=\vec{U} \cdot \vec{n}$ and $V_{n}=\vec{V} \cdot \vec{n}$ with $\tilde{r}_{0}=0.165 \mathrm{~m} / \mathrm{s}$ and $c_{f}=3.24 \mathrm{~s} / \mathrm{m}$. Then

$$
\frac{d \vec{R}}{d t}=\tilde{r}_{0}\left(1+c_{f} \vec{V} \cdot \vec{n}\right) \vec{n}
$$

If the fire front curve at any specified time $t$ is described by the vector function $(x(s, t), y(s, t))$, where $s$ is a parameter specifying the curve, then, the unit tangent vector can be written as

$$
\vec{\tau} \equiv \frac{1}{|\nabla \phi|}\left(\frac{\partial x}{\partial s} \vec{i}_{x}+\frac{\partial y}{\partial s} \vec{i}_{y}\right)
$$

where $|\nabla \phi|=\sqrt{(\partial x / \partial s)^{2}+(\partial y / \partial s)^{2}}$. The unit normal vector is

$$
\vec{n} \equiv \frac{1}{|\nabla \phi|}\left(-\frac{\partial y}{\partial s} \vec{i}_{x}+\frac{\partial x}{\partial s} \vec{i}_{y}\right)
$$

In component form, the equations can be written

$$
\begin{aligned}
& \frac{d x}{d t}=U_{n} n_{x} \\
& \frac{d y}{d t}=U_{n} n_{y}
\end{aligned}
$$

where

$$
U_{n} \equiv \tilde{r}_{0}\left(1+c_{f} \frac{(-\partial y / \partial s) V_{x}+(\partial x / \partial s) V_{y}}{\sqrt{(\partial x / \partial s)^{2}+(\partial y / \partial s)^{2}}}\right)
$$

\section{Eulerian Equations}

At this point, we turn to the development of the equations for the front propagation in Eulerian form. As in the book of Sethian [5], assume that the front is defined by the contour $\phi(x, y, t)=0$ of the front function $\phi$. Let $U_{x}, U_{y}$ be the components of the spread velocity normal to the front, $\vec{U}=U_{x} \vec{i}_{x}+U_{y} \vec{i}_{y}$.

If, as above, we take the parameter $s$ to be the distance along the surface $\phi(x, y, t)=0$ from a specified point along the front, then

$$
\left(\frac{\partial \phi}{\partial x}\right) \frac{d x}{d s}+\left(\frac{\partial \phi}{\partial y}\right) \frac{d y}{d s}=0
$$

so that $\frac{d x}{d s} \approx-\frac{\partial \phi}{\partial y}$ and $\frac{d y}{d s} \approx \frac{\partial \phi}{\partial x}$. Then, the unit tangent vector can be written

$$
\vec{\tau}=\frac{1}{|\nabla \phi|}\left(-\frac{\partial \phi}{\partial y} \vec{i}_{x}+\frac{\partial \phi}{\partial x} \vec{i}_{y}\right)
$$


while the unit normal vector becomes

$$
\vec{n}=\frac{1}{|\nabla \phi|}\left(\frac{\partial \phi}{\partial x} \vec{i}_{x}+\frac{\partial \phi}{\partial y} \vec{i}_{y}\right)
$$

where $|\nabla \phi|=\sqrt{(\partial \phi / \partial x)^{2}+(\partial \phi / \partial y)^{2}}$.

Write out the complete Eulerian equations:

$$
\frac{\partial \phi}{\partial t}+U_{x} \frac{\partial \phi}{\partial x}+U_{y} \frac{\partial \phi}{\partial y}=0
$$

where

$$
\begin{array}{ll}
U_{x}=\tilde{r}_{0}\left(1+c_{f} \vec{V} \cdot \vec{n}\right) n_{x} & \equiv f(|\vec{V}|, \vec{V} \cdot \vec{n} /|\vec{V}|) n_{x}, \\
U_{y}=\tilde{r}_{0}\left(1+c_{f} \vec{V} \cdot \vec{n}\right) n_{y} & \equiv f(|\vec{V}|, \vec{V} \cdot \vec{n} /|\vec{V}|) n_{y} .
\end{array}
$$

The spread vector, $\left(U_{x}, U_{y}\right)$, is normal to the fire front and has a magnitude equal to the "speed function" defined by Wolff and Fendell [6], For the examples shown below, the speed function is taken to be $\tilde{r}_{0}\left(1+c_{f} \vec{V} \cdot \vec{n}\right)$. Generally, the speed function depends upon the wind speed $|\vec{V}|$ and the angle $\theta$ between the normal to the front and the wind velocity: $\vec{V} \cdot \vec{n} /|\vec{V}| \equiv \cos \theta$.

\section{Level Set Numerical Method}

In this section we present our method for solving the level set equation (9). The method is generally second-order in space and time but uses flux limiting schemes to preserve monotonicity of the scalar field.

The 2D spatial domain is $L_{x} \times L_{y}$ with uniform grid spacings $\Delta x$ and $\Delta y$ in the $x$ and $y$ directions, respectively. The scalar $\phi(i \Delta x, j \Delta y, n \Delta t)$ and the speed function (i.e. rate of spread) $\vec{U}(i \Delta x, j \Delta y, n \Delta t)$ are node centered: $i=0: N_{x}, j=0: N_{y}, n$ is time step index. No flux, or zero gradient, boundary conditions are applied along each of the boundaries. The initial condition for $\phi$ is arbitrary, except that there must be some initial curve or curves representing the fire front initially for which $\phi(x, y, t=0)=0$.

In general, the speed function depends on the scalar gradient at the node location. However, to preserve monotonicity of the scalar field, the scalar gradient is obtained from a flux limiter which is based on the speed function. We avoid this circular problem by computing the speed function using a scalar gradient obtained from a central difference (no limiter). This speed function is then used in the PDE and also in determining the limited scalar gradient for the PDE. The basic steps of the numerical procedure are as follows:

Step 1: Given the scalar field at time $t^{n}$, the first step in the numerical procedure is to compute the node-centered scalar gradient by a central difference,

$$
\begin{aligned}
& \left(\frac{\delta \phi}{\delta x}\right)_{i, j}^{n}=\frac{\phi_{i+1, j}^{n}-\phi_{i-1, j}^{n}}{2 \Delta x} \\
& \left(\frac{\delta \phi}{\delta y}\right)_{i, j}^{n}=\frac{\phi_{i, j+1}^{n}-\phi_{i, j-1}^{n}}{2 \Delta y}
\end{aligned}
$$

where $\delta / \delta x$, for example, represents the numerical approximation to the partial derivative. In what follows we will drop the time stamp until discussion of the time integration scheme.

Step 2: Use the differences obtained in Step 1 to determine the fire front unit normal vector (8) and compute the speed function via (10).

Step 3: Given the front velocity at each node from Step 2, $\vec{U}_{i, j}^{n}$, determine the monotonicity preserving scalar gradient for the PDE. The limiter scheme choices in our level set code (in order of increasing accuracy) are: (1) first-order upwinding, (2) minmod, and (3) Superbee [10]. As an example of how the flux limiter is implemented, consider the computation of the limited scalar gradient in the $x$ direction. 
We imagine a cell of width $\Delta x$ centered at the node location. The gradient is determined from a central difference of the scalar face values for the cell,

$$
\frac{\delta \phi}{\delta x}=\frac{\phi_{\text {east }}-\phi_{\text {west }}}{\Delta x}
$$

The scalar face values are determined from the flux limiter scheme. In this example, let us compute the value for the east face $\left(i+\frac{1}{2}\right)$ and assume that $U_{x}>0$. In the limiter scheme, the first step is to compute the local and upwind data variations,

$$
\begin{aligned}
\Delta_{l o c} & =\phi_{i+1, j}-\phi_{i, j}, \\
\Delta_{u p} & =\phi_{i, j}-\phi_{i-1, j} .
\end{aligned}
$$

These values are used to form the local data ratio,

$$
r=\frac{\Delta_{u p}}{\Delta_{l o c}}
$$

In practice, we set $r=0$ if $\Delta_{l o c}=0$. The face value is now simply determined from

$$
\phi_{\text {east }}=\phi_{i, j}+\frac{1}{2} B\left(\phi_{i+1, j}-\phi_{i, j}\right),
$$

where $B$ is the flux limiter function evaluated from one of the following, Toro [10]:

$$
\begin{array}{ll}
B(r)=0 & \text { first-order } \\
B(r)=\max (0, \min (1, r)) & \text { minmod, } \\
B(r)=\max (0, \min (2 r, 1), \min (r, 2)) & \text { Superbee. }
\end{array}
$$

Note that when $B=1$, the scheme recovers second-order central differencing.

Step 4: Time integration. We have now discussed all the details associated with computing the right hand side (RHS) of the scalar partial differential equation (PDE). To advance the field in time, we use a second-order Runge-Kutta scheme, Gottlieb [11]. This scheme is simply a linear combination of two Forward Euler steps. Let $F(\phi)=\vec{U} \cdot \bar{\nabla} \phi$ denote the advective terms of the PDE obtained via Steps 2 and 3 above; $\bar{\nabla}$ denotes the limited discrete gradient operator. The time integration proceeds as follows:

$$
\begin{aligned}
\phi_{i, j}^{*} & =\phi_{i, j}^{n}-\Delta t F_{i, j}\left(\phi^{n}\right), \\
\phi_{i, j}^{n+1} & =\frac{1}{2} \phi_{i, j}^{n}+\frac{1}{2}\left(\phi_{i, j}^{*}-\Delta t F_{i, j}\left(\phi^{*}\right)\right) .
\end{aligned}
$$

The fire front is obtained from the zero level crossing of the scalar field. In Matlab this level set is easily visualized using the contour utility.

\section{Initial Results using the Level-Set Method}

The speed function $\tilde{f}(V, \theta)$ and the assumption that the front moves normal to itself into the unburned fuel determine how the fire front will propagate, Sethian [5], Fendell and Wolff [6], and Rehm and Mell [4]. A general form for the speed function has been suggested by Fendell and Wolff [6]. It appears that the speed function can be specified by three measurements: (1) the head-fire speed (measured in the direction of the wind), (2) the flank speed (measured in the cross-wind direction), and (3) the tail speed (measured opposite to the wind speed). However, neither the form nor the parameters used in this form are established generally for wildland fires, in large part because the field measurements are difficult to make. Earlier studies, Rehm [2] and Rehm and Mell [4], used a speed function appropriate for Australian grass fires $\tilde{r}_{0}\left(1+c_{f} \vec{V} \cdot \vec{n}\right)$, since both the form and the parameters were found by Cheney et al. [9] to match reasonably well field measurements. Some results reported here use this speed function for the simulations, while others use a simplified version of the more general speed function of Fendell and colleagues [6],[7]. 
The two speed functions used in the simulations below are:

$$
\tilde{f}(V, \theta)=\tilde{r}_{0}\left(1+c_{f} \vec{V} \cdot \vec{n}\right),
$$

and the simplified speed function of Mallet et al [7], expressed in our notation as:

$$
\begin{aligned}
& \tilde{f}(V, \theta)=\tilde{r}_{0}\left(1+c_{f} \sqrt{V} \cos ^{n} \theta\right) \quad|\theta| \leq \pi / 2, \\
& \tilde{f}(V, \theta)=\tilde{r}_{0}(\alpha+(1-\alpha) \sin \theta) \quad \pi / 2<|\theta| \leq \pi \text {. }
\end{aligned}
$$

Here, $n$ and $\alpha$ are parameters, taken to be $n=1.5$ and $\alpha=0.5$ for the simulations shown in Figures 1 and 2. The remaining simulations, shown in Figures 3, 4 and 5, used the speed function given in Eq. (20).

We now discuss some simulations that were carried out to compare the Lagrangian and Eulerian methodologies. The initial comparison was for the trivial case of a straight-line front in a wind blowing perpendicular to the front with periodic boundary conditions in the cross-wind direction. The spread function was for Australian grass, Eq.(20), with a wind speed of $3 \mathrm{~m} / \mathrm{s}$. For the Lagrangian formulation with the MOL solution procedure, the propagation speed was found to agree with the value calculated from Eq. (20) to within one tenth of one percent, whereas the Eulerian/level-set method was found to agree with the value calculated by Eq. (18) to approximately 3.5 per cent $\left(N_{x}=N_{y}=30\right)$ and 3 percent $\left(N_{x}=N_{y}=40\right)$.

Figures 1 and 2 below show comparisons between the two methods for the simulation of fire front propagation for a nontrivial case, an initially circular fire front blown by a uniform wind of $3 \mathrm{~m} / \mathrm{s}$ in the $y$ direction. The initial front is taken to have a radius of $10 \mathrm{~m}$, centered at $x=0, y=50$. The speed function given in (21) with $\tilde{r}_{0}=0.165 \mathrm{~m} / \mathrm{s}, c_{f}=3.24 \mathrm{~s} / \mathrm{m}, n=1.5, \alpha=0.5$, is used. In Figure 1 , the front is shown at five equal time intervals $6 \mathrm{~s}$ apart. The Eulerian formulation, using the level set solution method, is shown on the left; at each time, the zero contour is obtained by interpolation from the Eulerian solution. For comparison, the Lagrangian formulation using the method of lines solution of the same problem is shown on the right. For this method, successive Lagrangian nodes have simply been joined by straight lines; therefore, the fire front in the windward direction gradually loses its smooth appearance as time increases. (A second or higher order interpolation of the front between Lagrangian nodes would provide a smoother contour with better visual agreement.)

Each simulation requires the selection of several parameters to carry out the simulation. For example, the Eulerian formulation requires the simulation domain to be discretized in both the $x$ - and the $y$ directions, and boundary conditions along each of the edges of this domain must be specified. For the case shown in Figures 1 and 2, a domain $100 \mathrm{~m}$ on a side $\left(L_{x}=L_{y}=100 \mathrm{~m}\right.$ with $0 \leq y \leq L_{y},-L_{x} / 2 \leq$ $\left.x \leq L_{x} / 2\right)$ was considered, with the discretization being 30 nodes $\left(N_{x}=N_{y}=30\right)$ in each direction. Alternately, the Lagrangian formulation requires that the initial fire front be discretized periodically along its circumference. For the case shown in Figures 1 and 2, 120 nodes were used along the circumference.

Variation of any of the parameters noted above can cause significant differences in the simulations, making detailed comparisons between simulation results, as well as simulation efficiencies, difficult. All simulations were carried out using Mathematica [8]. The simulations for a given resolution using the level set method generally required more cpu time than did the MOL solutions with comparable resolution. However, the parameters for each type of simulation were not varied systematically to determine cpu time dependencies. Also, we did not attempt to determine the expected cpu time differences between the two methodologies.

In Figure 2, the fire fronts from each simulation are compared directly. In the first frame of Figure 2, the initial fire fronts are shown together, while the second compares the fronts after $6 \mathrm{~s}$, the third frame after $12 \mathrm{~s}$, etc. (Note that the fire fronts contours obtained from each simulation have not been smoothed.) As a reference, during the $30 \mathrm{~s}$ of simulation time, the head velocity is expected to be $1.09 \mathrm{~m} / \mathrm{s}$, for a wind speed of $3 \mathrm{~m} / \mathrm{s}$. After $30 \mathrm{~s}$, the head of the fire should be at $x=0, y=92.7 \mathrm{~m}$. The level-set simulation determines the head location to be at $y=93.9 \mathrm{~m}$ while the Lagrangian, MOL simulation, determines the head location to be at $y=92.0 \mathrm{~m}$. Both simulations seem to do a reasonable job quantitatively of determining the fire head location.

In the next two figures, capabilities touted by the advocates of the level-set methodology are illustrated. The merger of two fire fronts is illustrated in Figure 3, and in Figure 4, fronts are shown spreading to form an island or pocket of unburned fuel, that is subsequently consumed. Figure 3 shows four frames 
from a level-set simulation of the merger of two fire fronts, one a straight-line front progressing in the positive $y$-direction, and the other generated by a growing spot fire ahead of the straight-line front. Figure 4 shows four frames from a level-set simulation of the merger of three fire fronts, producing an island or a pocket of unburned fuel, which is subsequently consumed.

The ability to qualitatively describe each of these situations arises naturally from the LSM and is generally regarded as great advantage of the method. Although the behavior of the resulting fronts seems physically realistic, there is no assurance that this appealing qualitative behavior is in fact quantitatively correct. Furthermore, there is little prospect for quantitative evaluation of the behavior.

On the other hand, in the Lagrangian formulation, an algorithm that specifies either a front merger or the development of a fuel pocket behind a front under a variety of conditions is a daunting conceptual and programming task. To our knowledge, no physical description of a fire-front merger exists, nor do measurements of the phenomenon, for example. Furthermore, even if there were a valid theoretical description of the phenomena, programming the behavior of complex mergings of fronts would be tedious.

Finally, in Figure 5, a line fire front is generated from the merger of three initial circular fires that have been allowed to propagate under the influence of a $3 \mathrm{~m} / \mathrm{s}$ ambient wind in the y-direction. Each initially circular front grows preferentially in the $y$-direction (head direction), but also grows in the $x$ - or flank direction until the three separate fronts merge into a single, scalloped front. As noted before, the difference between the head and flank ROS is dependent upon the speed function used in the simulation.

\section{Discussion*}

Equation (9) determines the advance with time of the function $\phi(x, y, t)$, whose level set $\phi=0$, represents the fire front. Note that the only quantity that has physical meaning is the location of the curve $\phi(x, y, t)=$ 0 . This fact underlies both the attractiveness of the level set approach and the issues discussed below.

In order to solve this equation on a finite domain, boundary conditions must be specified. Moreover, the solution obtained must depend at least to some degree on the boundary conditions chosen. However, since the function $\phi$ has no meaning away from the front, the boundary conditions chosen cannot be justified by appeal to physical principles related to fire dynamics. In sufficiently simple situations, solutions obtained using this technique can be compared with those obtained by directly tracking the location of the front (the "Lagrangian" technique) as discussed above. Agreement between the two approaches is then offered as evidence of the correctness of the level set technique, as done in the previous section of this report. However, there is no guarantee that boundary conditions that work for a single isolated fire front are necessarily correct in more complex situations.

Another uncertainty arises when the solution obtained describes the merging and creation of "islands" of unburned fuel, as shown in the examples above. The fact that solutions of this type can be readily obtained using level set techniques is often cited as an indication of the superiority of the method. This may be true, but it rests on the assumption that the merging process, where the direction normal to the front is essentially undefined, is correctly described. Since front tracking models are by definition undefined in such regions, this conclusion can only be justified if an analysis based on a more complete, physically based model can be analyzed in some asymptotic limit to produce a similar result. This does not seem to have been done for fire fronts. Such an analysis would doubtless be difficult to perform, since it is far from clear what more detailed model should be employed as the starting point for any such study.

At this point in time, a reasonable assessment would be that front tracking and level set approaches result in two different models. For sufficiently simple scenarios, they are equivalent. However, the level set approach clearly offers much greater generality than front tracking. In the absence of definitive analytical or experimental data, this would clearly be the preferred approach, once the role of boundary conditions has been properly addressed. It may well be that by choosing a sufficiently large computational domain, the issue of boundary conditions can be finessed. If so, then the level set model can stand on its own as a simplified physics based approach for fire spread over larger domains.

* This discussion of the level set approach was prepared by Dr. Howard R. Baum. 


\section{References}

1. R.G. Rehm and D.D. Evans, "Physics - Based Modeling of Wildland - Urban Interface Fires," in "Remote Sensing and Modeling Applications to Wildland Fires," a volume Edited by John Qu in Springer's Geosciences Series. To appear 2009.

2. Rehm, Ronald G., "The Effects of Winds from Burning Structures on Ground-Fire Propagation at the Wildland-Urban Interface," Combustion Theory and Modelling, Volume 12, Issue 3, 2008, Pages 477-496.

3. Mell, William E., Samuel L. Manzello, Alexander Maranghides and Ronald G. Rehm, "WildandUrban-Interface Fires: Overview and Research Needs," International Journal of Wildland Fire. Invited paper, under review.

4. Rehm, Ronald G. and William (Ruddy) Mell, "A Simple Model for Wind Effects of Burning Structures and Topography on WUI Surface-Fire Propagation," accepted for publication in the International Journal of Wildland Fire.

5. Sethian, J.A., Level Set Methods and Fast Marching Methods: Evolving Interfaces in Computational Geometry, Fluid Dynamics, Computer Vision, and Material Science, Cambridge Monographs on Applied and Computational Mathematics, Cambridge University Press, 1999.

6. Fendell, F.E. and M.F. Wolff, (2001)"Wind-Aided Fire Spread, Forest Fires, Behavior and Ecological Effects," Edward A. Johnson and Kiyoko Miyanishi (eds) Chapter 6, pp171223.

7. Mallet, V., D.E. Keyes and F.E. Fendell, (14 October 2007) "Modeling Wildland Fire Propagation with Level Set Methods," 22 pp.

8. Mathematica Version 6 software: see http://www.wolfram.com/

9. Cheney, N.P., J.S. Gould and W.R. Catchpole (1998) "Prediction of Fire Spread in Grasslands," International Journal of Wildland Fire, 8, 1-13.

10. Toro, E.F., "Reimann Solvers and Numerical Methods for Fluid Dynamics: A Practical Introduction," Second Edition, Springer (1999).

11. Gottlieb, S., C.W. Shu, E. Tadmor, "Strong Stability-Preserving High-order Time Discretization Methods," SIAM Review, Vol. 43, Number 1, pages 89-112, (2001). 
Y

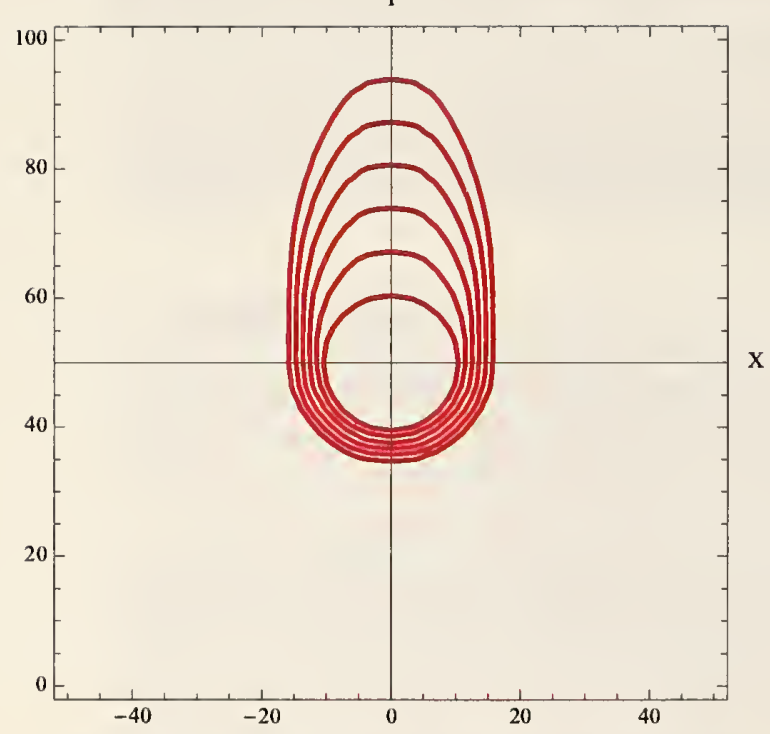

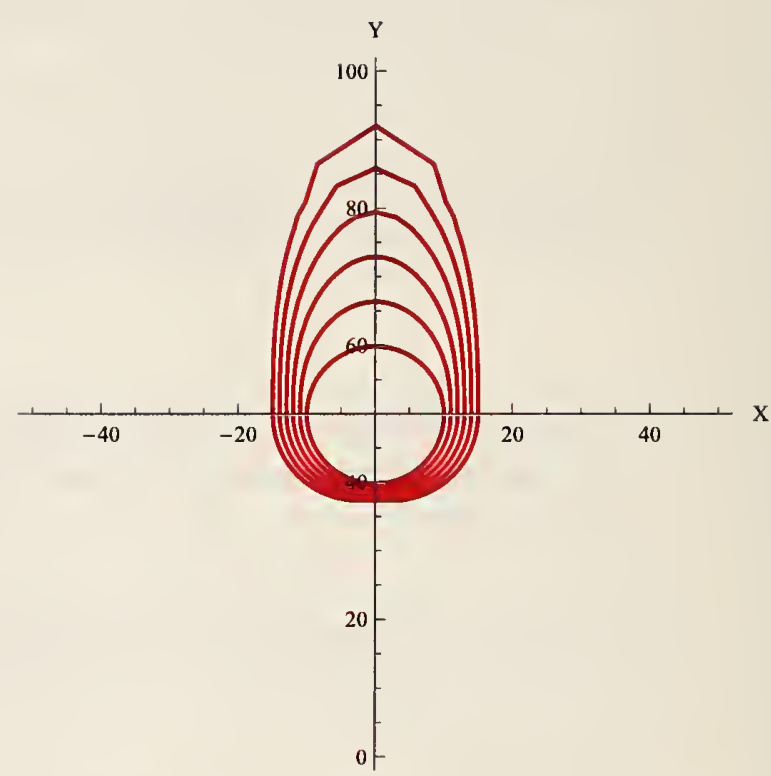

Figure 1. LEFT: The progression of a wind-blown fire front from an initially circular fire is shown. The model is formulated as a two-dimensional Eulerian partial differential equation (PDE) and solved with a level-set methodology. For initial conditions, the front is taken to be circular with a radius of 10 $\mathbf{m}$, centered at $x=0, y=50$. The scenario shows what might be expected during the growth of a spot fire when a steady wind is blowing in the positive y-direction.RIGHT: The same scenario as shown on the left, except that these results were obtained using the Lagrangian formulation for the front progression and solving the resulting ordinary differential equations (ODEs) by the method of lines (MOL). 


\begin{tabular}{|l|l|}
\hline 0 & 0 \\
\hline 0 & 0 \\
\hline 0 & 0 \\
\hline
\end{tabular}



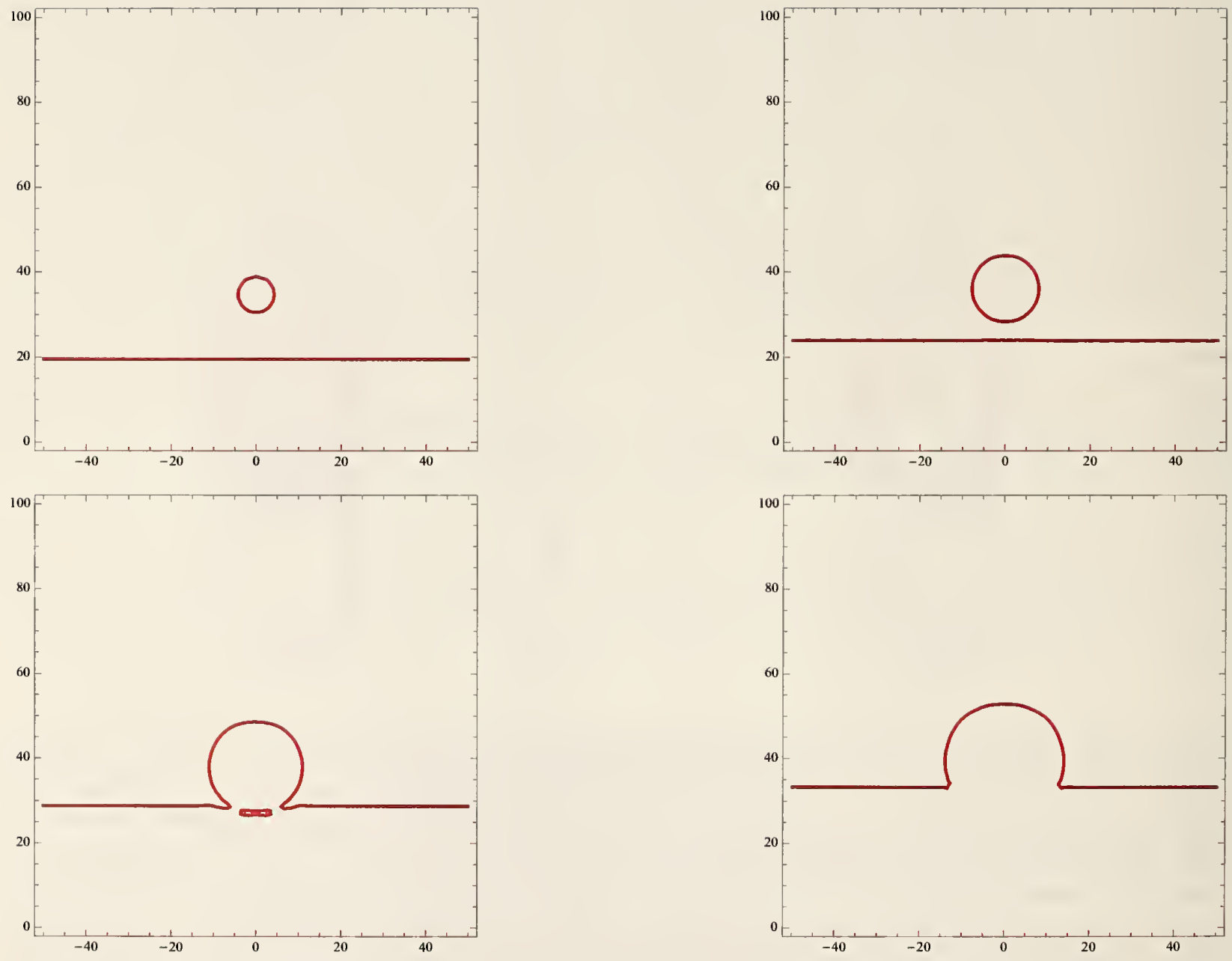

Figure 3. Four frames from a level-set simulation of the merger of two fire fronts, one a straight-line front progressing normal to itself in the positive $y$-direction, and the other generated from a spot fire ahead of the line fire. The order of the frames is upper left first, upper right second, lower left third and lower right last. 

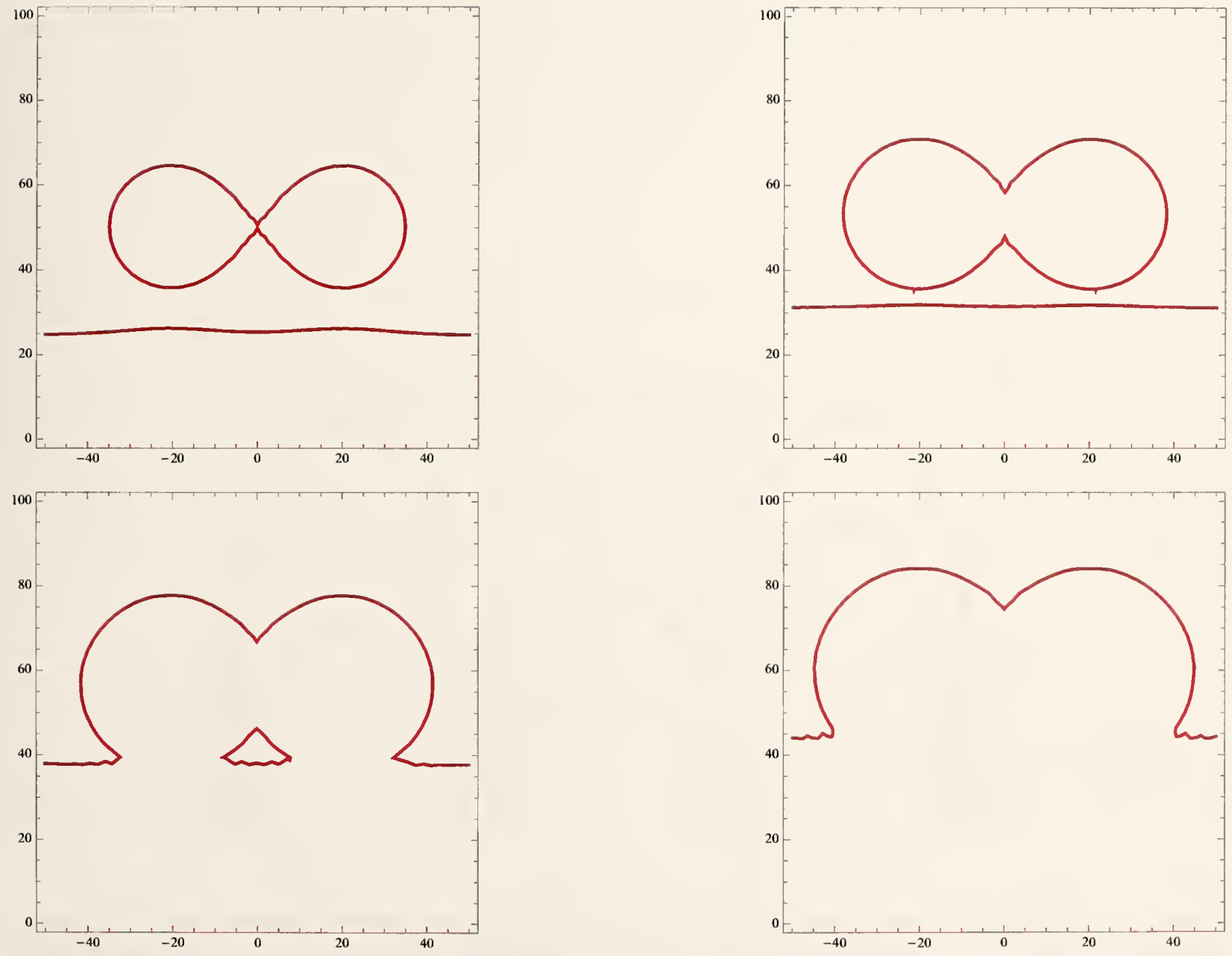

Figure 4. Four frames from a level-set simulation of the merger of three fire fronts, producing an island or a pocket of unburned fuel, which is subsequently consumed. 


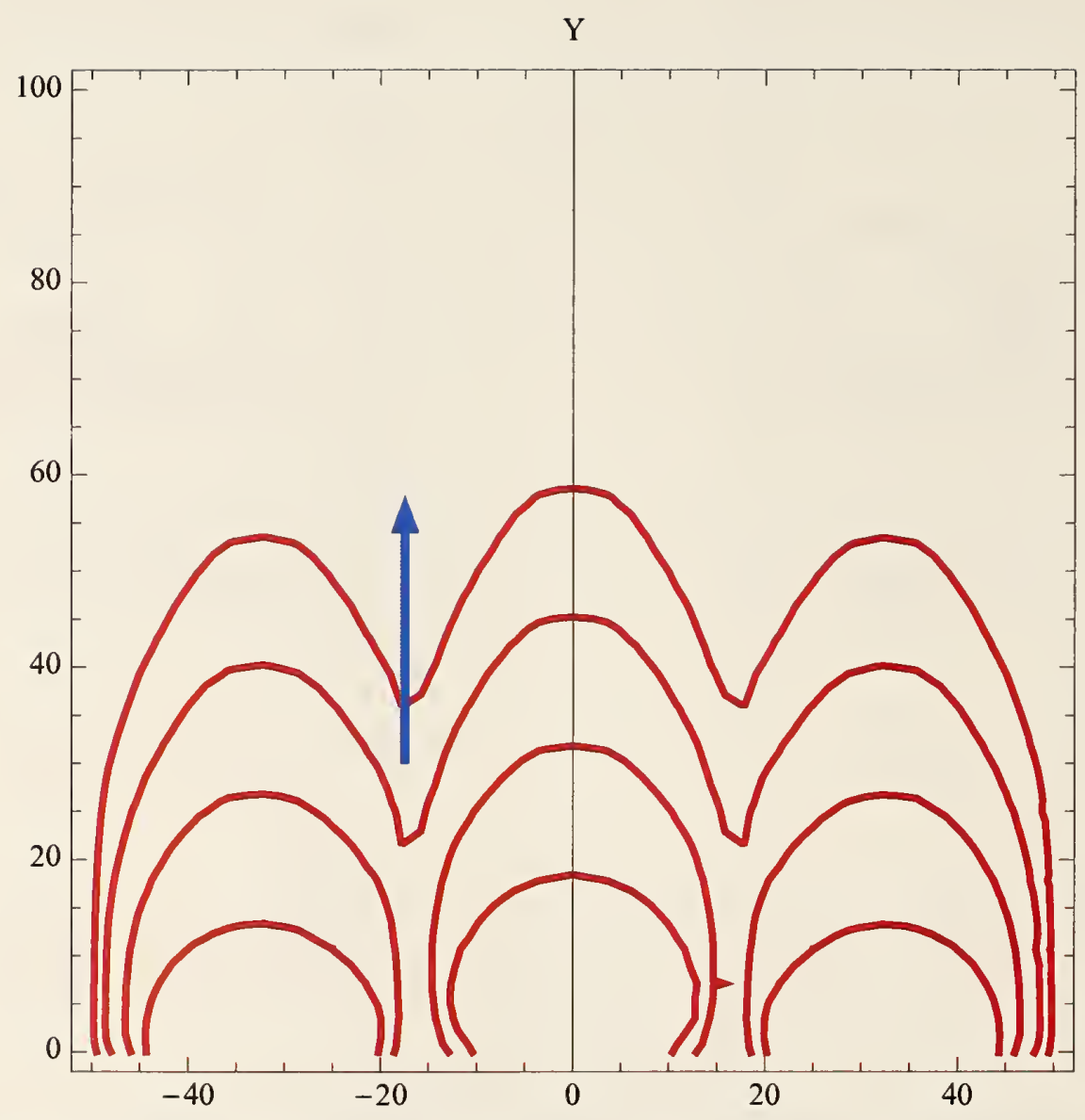

Figure 5. A wind-driven fire front shown at four times during the level-set simulation. The front is initially generated by 3 circular fire fronts placed symmetrically with respect to $x$. The head of each circular front propagates in the direction of the wind, while the flanks propagate laterally and merge into a single front. For this simulation, the ambient wind is $3 \mathrm{~m} / \mathrm{s}$ in the $y$-direction. 

